\title{
Device Observer Manufacturer
}

National Cancer Institute

\section{Source}

National Cancer Institute. Device Observer Manufacturer. NCI Thesaurus. Code C69251.

The manufacturer of an observation device. 